\title{
Molecular Dynamics simulations of Couette flow
}

Juan A. Martín, Julio R. Meneghini and Vassilis Theofilis

\begin{abstract}
In this work, the first steps towards developing a continuum-molecular coupled simulation technique are presented, for the purpose of computing macroscopic systems of confined fluids. The idea is to compute the interface wall-fluid by Molecular Dynamics (MD) simulations, where Lennard-Jones potential (and others) have been employed for the molecular interactions, so the usual no slip boundary condition is not specified. Instead, a shear rate can be imposed at the wall, which allows the calculation of wall material properties by means of an iterative method. The remaining fluid region will be computed by a spectral hp method. We present MD simulations of a Couette flow, and the results of the developed boundary conditions from the wall fluid interaction.
\end{abstract}

\section{Introduction}

In the continuum hypothesis, the analysis of macroscopic flows over a specific solid geometry needs to impose boundary conditions for the velocity of the fluid at the solid wall [1]. It is generally used the no slip condition at the boundaries, which specifies that the velocity of the fluid relative to the wall is zero at the solid-fluid interface. At the molecular scale, the description of nano and micro-fluids has been

Juan A. Martín

ETSI Aeronáuticos, Universidad Politécnica de Madrid

Pza. Cardenal Cisneros 3, 28040 Madrid, Spain, e-mail: jamartin@fmetsia.upm.es

Julio R. Meneghini

Núcleo de Dinâmica e Fluidos, Departmento de Engenharia Mecânica, Escola Politécnica da Universidade de São Paulo, Av. Professor Mello Moraes 2231, 05508-030 São Paulo, Brazil, e-mail: jmeneg@usp.br

Vassilis Theofilis

ETSI Aeronáuticos, Universidad Politécnica de Madrid

Pza. Cardenal Cisneros 3, 28040 Madrid, Spain, e-mail: vassilis@aero.upm.es 
achieved by MD simulations. They are capable of accurately describing the dynamics of the wall-fluid interaction, and also can take into account the effect of wall material properties and the morphology of the surface. MD simulations consist of solving the Newton's equations of motion for a system of interacting discrete molecules, in order to follow its evolution at the molecular scale $[2,3,4]$. The obtained microscopic results by this method are usually averaged by statistical mechanics, in order to provide macroscopic properties. In this case, there is no need to make any assumptions about the boundary conditions (apart from using particles with prescribed velocity) at the interface wall-fluid, instead, they will be given as an output of the numerical model, and then, will be translated to the macroscopic scale.

\section{Molecular Dynamics simulation model}

The model system consists of a monoatomic fluid confined between two atomistic walls. The top wall was set to be moving in parallel to the bottom wall, that remains stationary. With this configuration the steady-state of Couette flow is developed. All simulations included in this work were performed using the parallelized MD code LAMMPS [7].

The fluid molecules interact through the Lennard-Jones (LJ) potential

$$
V_{i j}(r)=4 \varepsilon_{i j}\left[\left(\frac{\sigma_{i j}}{r}\right)^{12}-\left(\frac{\sigma_{i j}}{r}\right)^{6}\right],
$$

in which $\varepsilon$ is the energy scale, $\sigma$ is the length scale, the value of $r$ where the potential is zero, and the indexes represent the interaction between the atom $i$ and the atom $j$. The results obtained are expressed in terms of these values, and will be given in reduced dimensionless units. A cutoff distance $r_{c}$ is applied $\left(r_{c}=1.12 \sigma\right)$, beyond which the value of the potential is set to zero. The equation of motion for atom $i$ with mass $m_{i}$ is

$$
m_{i} \frac{d^{2} \mathbf{r}_{i}}{d t^{2}}=\mathbf{F}_{i}=-\sum_{i \neq j} \frac{\partial V_{i j}}{\partial \mathbf{r}_{i}}
$$

The temperature of the fluid was controlled by applying a thermostat to the fluid atoms, a very simple re-scaling of the velocities, in order to maintain the target temperature of the system, set to $T_{0}=1.0$ in LJ units.

The equations of motions were solved using a velocity-Verlet algorithm with a time step of $0.001 \mathrm{LJ}$ time units, $\left(\tau=\sqrt{m \sigma^{2}}\right)$ and the top wall was given a sliding velocity of $U_{0}=3.0$ at the start of the simulation, time $t=0$. Periodic boundary conditions in the horizontal coordinate are imposed. The solid wall thickness was considered reviewing the values found in the literature, from 5 to 10 rows of atoms in $[9,11,12]$, finding no differences when our simulations were carried out. Finally, 8 rows of atoms were selected to represent the wall. 


\section{MD simulations results}

Different simulations were performed, for two-dimensional domains. The biggest one with a size of $600 \times 200$ length units in the horizontal and vertical directions, containing a total number of atoms simulated of 250,000 . With our computing capabilities, this simulation takes 2 weeks to completely develop the Couette flow. Regarding the CPU cost, smaller simulations (6,000 atoms) were used to analyze the LJ parameters effect. The density of the lattice was $\rho=0.8$.

The planar Couette flow with no body forces can be described by the incompressible Navier-Stokes equations [5], which provide the one dimensional solution to the geometry presented. The $x$-component of the momentum equation is given by

$$
\rho \frac{\partial u}{\partial t}=\mu \frac{\partial^{2} u}{\partial y^{2}}
$$

with boundary and initial conditions

$$
u(0, t)=0, \quad u(L, t)=U_{0}, \quad u(y, 0)=0,
$$

where the boundaries between wall and the liquid are $y=0$ at the bottom, and $y=L$ at the top. The solution of eq. (3) can be expressed by Fourier series [6] and it is used to compare MD results with the analytic solution.

In a first case, the MD simulations were performed using the same interaction between fluid atoms and wall atoms, $\varepsilon_{f, w},(f$ stands for fluid and $w$ stands for wall $)$ than the interaction between fluid atoms themselves, $\varepsilon_{f, f}$. In other words, the system is $\varepsilon_{w, f}=\varepsilon_{f, f}$. The velocity profile obtained by MD simulations is compared with the analytic solution in Fig. 1. The streamwise velocity is spatial and time averaged in $N$ horizontal slices along the vertical coordinate. The continuum analytic profile is calculated by eq. 3 with $\mu=1.6$, value taken from [8] when $\rho=0.8$ and $T=1.0$.

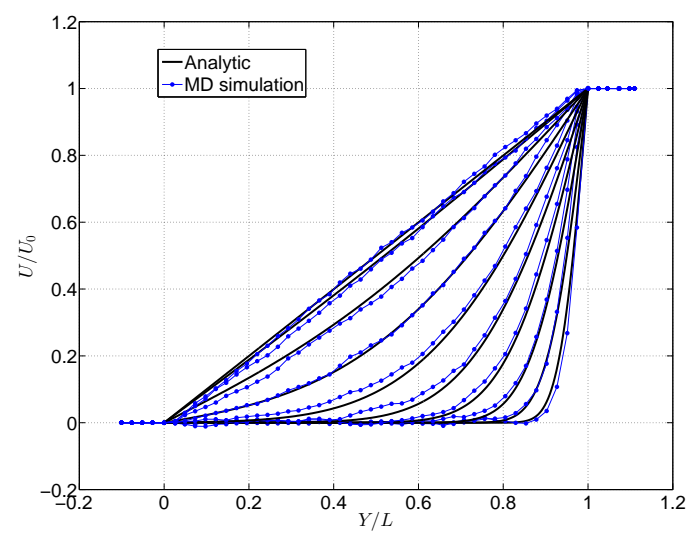

Fig. 1 Streamwise velocity profile at times $\tau=2^{n}$, for $n=3,4,5, \ldots 12$ from right to left. The black line represents the analytical solution and the average of streamwise velocity profile obtained by MD simulations is in blue dots, for different vertical locations. Simulation with 250, 000 atoms 
As observed in Fig. 1, the start-up and steady Couette flow is well captured with the molecular model used, and it is capable of reproducing the different stages of the flow development, recovering the expected behavior from continuum hydrodynamics. The differences observed during the transient process might be due to the impossibility of imposing a constant pressure in the MD simulations.

A second round of simulations were launched, modifying the interaction between fluid and walls. In that case, the wall has an interaction with the fluid characterized by $\varepsilon_{w, f}=0.6 \varepsilon_{f, f}$, and also the atomistic walls have a reduce density of $\rho_{w}=2.8 \rho_{f}$. These values were chosen looking into [9]. The steady-state velocity profile obtained can be appreciated in Fig. 2 (a), where a slip at the solid-fluid interface is evident. This result is coherent with that reported in [9] and [13], and the simulations were performed with a geometry of $120 \times 40$ length units in the horizontal and vertical directions, accounting 6,000 atoms.

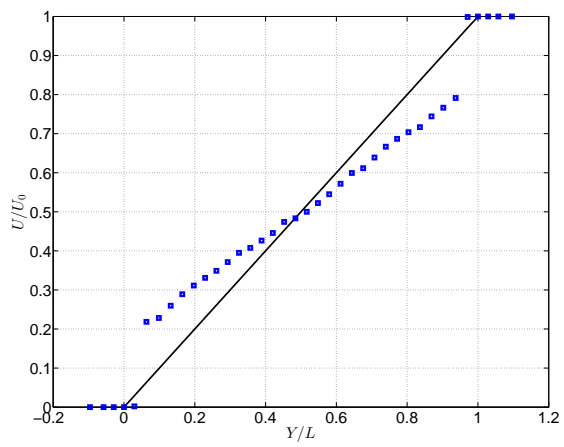

(a)

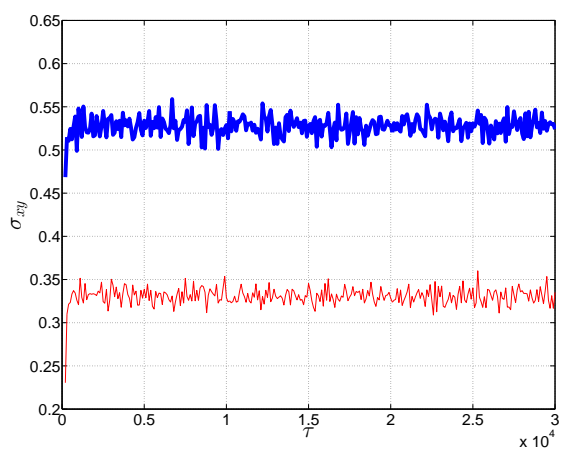

(b)

Fig. 2 (a) In blue squares, the steady state flow profile for a system with $\varepsilon_{w, f}=0.6 \varepsilon_{f, f}$, and $\rho_{w}=2.8 \rho_{f}$. The black line indicates the steady Couette flow. (b) Shear stress evolution. Thick blue line represents $\varepsilon_{w, f}=\varepsilon_{f, f}$ and $\rho_{w}=\rho_{f}$, while thin red line stands for $\varepsilon_{w, f}=0.6 \varepsilon_{f, f}$ and $\rho_{w}=2.8 \rho_{f}$. Both simulations with 6,000 atoms

It is also interesting to analyze the results concerning the shear stress at the wall, measuring the output shear force on the walls divided by the walls surface. Its time evolution is represented in Fig. 2 (b). As can be appreciated, the values of the shear stress (unlike the velocity values) exhibit a wide fluctuation, so very long simulation times must be considered to statistically obtain a representative value. From Fig. 2 (b), a reduction of the shear stress is observed for the simulation where interfacial slip is developed.

The main application of these simulations consists of the possibility of representing the different interfacial scenarios provided by textured or coated surfaces, which can consider the properties of the wall material, and can take into account the dynamics of the interfacial region. So, a tool to design low friction drag can be developed from this simulation, looking for the interaction between wall and fluid 
which can provide the drag force level required for a particular application (such as oil pumping in petrol enterprises).

\section{Conclusions}

MD simulations have the drawback of the heavy computational load required, which limits the simulations to a length scale of tens of nano-meters. So, in order to develop a useful simulation tool, these results can be used towards developing boundary conditions that will be applied in a continuum model. These integral boundary conditions will substitute the usual no slip condition by the velocity profile or shear stress obtained in this stage of the simulation, to elaborate a hybrid method which can combine both, molecular and continuum simulations in order to accurately describe the wall-fluid interaction and also represent the rest of the bulk fluid with a remarkable reduction of the computational cost.

In this work, the first steps to develop a code that includes Molecular Dynamics simulations and continuum hypothesis is developed. The canonical problem of Couette flow has been used to validate the numerical technique, obtaining very good agreement with the results given by MD simulations. The dynamics at the molecular scale plays a crucial role when determining the interaction between fluid and solid, which can define the level of shear stress at the wall. Therefore, the design of the wall surface can be achieved by MD simulations, and used as a passive way to reduce skin-friction drag, and to delay transition to turbulence.

The high computational cost of MD simulations forces us to combine this method with a continuum hypothesis which can provide a way to simulate a bulk fluid around a solid. This technique will take accurately into account the influence of the material properties, the texture of the surface or the coatings applied to the surface.

\section{Future and on-going work}

Currently, the main efforts are focused on the development of an iterative method to calculate potential parameters from the shear stress required at wall, in order to obtain the surface properties that best suit particular applications. Also, although the flow viscosity is tabulated in several works (see e.g. [8]), it will be calculated as a part of the simulation, to have an independent numerical technique. Likewise, LJ parameters are being matched with the Knudsen number, solving eq. (3) with slip boundary conditions, to provide more physical meaning. Finally, some complex flows are being considered, such as an open cavity Fig. 3 (a), or the flow over textured surfaces Fig. 3 (b). 


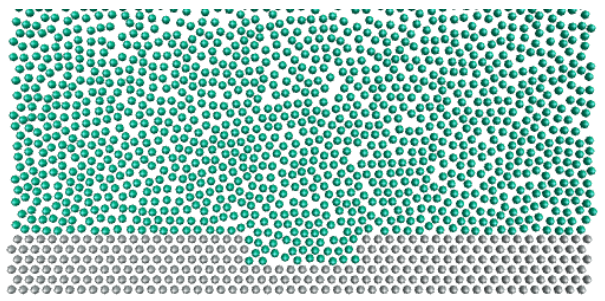

(a)

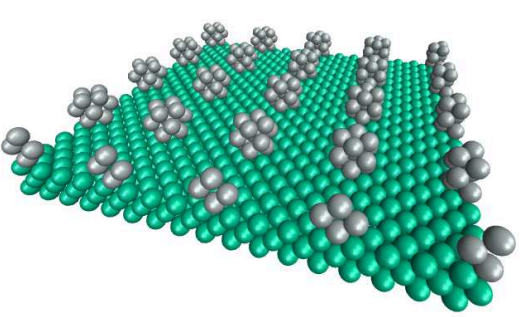

(b)

Fig. 3 Sketches of the ongoing work, (a) open cavity and (b) textured surface.

\section{Acknowledgments}

The support of the Marie Curie Grant PIRSES-GA-2009-247651 FP7-PEOPLEIRSES: ICOMASEF Instability and Control of Massively Separated Flows is gratefully acknowledged. We also would like to gratefully thank to Roberto Martins de Souza and Eleir Mundim Bortoleto for their help and guidance with the simulation and visualization software at our first steps into MD simulations.

\section{References}

1. G. K. Batchelor, Introduction to Fluid Dynamics, (Cambridge University Press, Cambridge, UK, 2000), pp. 60

2. J. M. Haile, Molecular Dynamics simulations, elementary methods, (John Wiley \& Sons, New York, USA, 1992)

3. M. P. Allen and D. J. Tildesley, Computer simulation of liquids, (Oxford University Press, Oxford, UK, 1987)

4. D. C. Rapaport, The art of Molecular Dynamics simulations, (Cambridge University Press, Cambridge, UK, 1995)

5. H. Schlichting, Boundary Layer Theory, (Springer, New York, 2000), pp. 130

6. W. A. Strauss, Partial differential equations, (John Wiley \& Sons, New Jersey, 1992)

7. S. Plimpton, (1995) Fast Parallel Algorithms for Short-Range Molecular Dynamics. J Comp Phys 117:1-19

8. F. da Costa Silva et al., (2003) Shear Viscosity Calculated by Perturbation Theory and Molecular Dynamics for Dense Fluids. J Quantum Chem 95:79-87

9. P. A. Thompson and S. M. Troian, (1997) A general boundary condition for liquid flow at solid surfaces. Nature 389:6649 360-362

10. E. R. Smith et al, (2012) Control volume representation of Molecular Dynamics. Phys Rev E 85:056705

11. D.M. Heyes et al., (2012) Pressure dependence of confined liquid behavior subjected to boundary-driven shear. J Chem Phys 136:134705

12. E. R. Smith et al, (2012) Control volume representation of Molecular Dynamics. Phys Rev E 85:056705

13. M. Cieplak et al. (2001) Boundary conditions at a fluid-solid interface. Phis. Rev. Letters $86: 5$ 803-806 